\title{
Transição alimentar: problema comum à obesidade e à cárie dentária
}

\author{
Changing from breastfeeding to family feeding: a \\ common problem for both obesity and dental caries
}

Jefferson TRAEBERT ${ }^{1}$

Emília Addison Machado MOREIRA²

Vera Lúcia BOSCO${ }^{3}$

Izabel Cristina Santos ALMEIDA ${ }^{3}$

\section{RE S U M O}

A obesidade, as doenças dela decorrentes e a cárie dentária têm no hábito alimentar um importante componente etiológico comum. Assim, o objetivo deste artigo foi revisar aspectos gerais da obesidade e da cárie dentária, enfocando a transição alimentar como problema comum ao desenvolvimento de ambas. As conseqüências da obesidade para a saúde são muitas, variando desde um maior risco de morte prematura até diversos problemas não fatais, mas debilitadores da qualidade de vida dos indivíduos. Os hábitos alimentares relacionados à obesidade podem também determinar uma maior prevalência de cárie dentária já que tanto a quantidade de sacarose ingerida quanto a freqüência de ingestão são importantes fatores envolvidos em sua etiologia. Os profissionais de saúde devem auxiliar e conscientizar os pais sobre os efeitos imediatos dos hábitos alimentares inadequados sobre a saúde da criança e do adolescente, principalmente em relação à obesidade e à cárie dentária.

Termos de indexação: obesidade, cárie dentária, saúde bucal, infância, adolescente, nutrição da criança.

\section{A B S T R A C T}

The dietary habits are important common etiological factors for both obesity and dental caries. The objective of this article was to review general aspects of obesity and dental caries focusing on the changing from

\footnotetext{
1 Centro de Ciências da Saúde, Programa de Pós-Graduação em Odontologia. Campus Universitário, 88010-970, Trindade,

Florianópolis, SC, Brasil. Correspondência para/Correspondence to: J. TRAEBERT. E-mail: jtraebert@uol.com.br

2 Departamento de Nutrição; Programa de Pós-Graduação em Odontologia, Universidade Federal de Santa Catarina.

3 Departamento de Estomatologia; Programa de Pós-Graduação em Odontologia, Universidade Federal de Santa Catarina.
} 
breastfeeding to family feeding as an important common problem for the development of both diseases. Obesity has been defined as a nutritional disturbance with results in several correlated diseases. The obesity related dietary habits could result in an increment of dental caries because both the amount and the frequency of sucrose ingestion are important factors associated with the aetiology of the oral disease. Thus, health professionals should increase parent's awareness about the immediate effects of obesity upon child's and adolescent's both general and oral health.

Index terms: obesity, dental caries, oral health, childhood, adolescent, child nutrition.

\section{N T R O D U Ç Ã O}

A obesidade, as doenças dela decorrentes e a cárie dentária têm no hábito alimentar um importante componente etiológico comum.

Segundo a Organização Mundial de Saúde1 (OMS), a prevalência de sobrepeso e da obesidade está aumentando em diversas partes do mundo, em ritmo alarmante. A obesidade está em crescimento em adultos e em crianças e os problemas de saúde decorrentes podem aparecer somente em um futuro distante. Em muitos países em desenvolvimento, ela co-existe com a subnutrição, sendo mais comum em áreas urbanas ${ }^{2}$. As mulheres geralmente têm maiores níveis de obesidade: entretanto, os homens apresentam maiores níveis de sobrepeso ${ }^{3}$. A obesidade e o sobrepeso estão associados com doenças cardíacas, hipertensão arterial, osteoartrite, diabetes tipo 2 e alguns tipos de câncer, sendo o seu impacto maior na morbidade do que na mortalidade ${ }^{1,4,5}$.

O papel do ambiente e da família aparece de forma clara em relação à etiologia da obesidade infantil. O maior risco para a obesidade infantil é a obesidade dos pais. Uma criança tem $80 \%$ de chance de ser obesa quando ambos, pai e mãe, são obesos, e $40 \%$ quando tem um dos pais obeso. Quando nenhum dos pais é obeso a chance é reduzida a 7\% ${ }^{6}$.

No Brasil, dois estudos de base nacional representativos e comparáveis foram realizados em 1974/75 e 1989 e demonstraram aumento da obesidade em adultos em ambos os sexos. Entretanto, detectou-se maior aumento entre famílias de baixa renda. O país está mudando rapidamente de um problema de déficit para excesso alimentar?.

Dados de prevalência de obesidade infantil no Brasil são escassos, pelo fato de os estudos não utilizarem amostras representativas da população, sendo restritos faixas etárias e grupos populacionais específicos ${ }^{8}$. Um estudo de prevalência de sobrepeso e obesidade em crianças e adolescentes das Regiões Sudeste e Nordeste mostrou que o sobrepeso em adolescentes é maior no sexo feminino. Concluiu ainda que a prevalência de obesidade na faixa etária de 2-17 anos é menor na Região Nordeste e que não houve diferença estatisticamente significativa entre as Regiões Nordeste e Sudeste, nas faixas etárias de crianças menores de dois anos e de adolescentes maiores de 18 anos de idade 9 .

Estudos detalhados de risco relativo entre vários problemas de saúde associados à obesidade são limitados aos países industrializados. Estes estudos demonstram que pessoas obesas têm a chance aumentada em mais de três vezes, de sofrer de diabetes mellitus tipo 2, doenças renais, dislipidemia e apnéia durante o sono. Têm, ainda, a chance aumentada entre duas e três vezes de sofrer de doenças cardiovasculares e osteoartrites e de uma a duas vezes de sofrer de certos tipos de câncer e anormalidades hormonais relacionadas à reprodução. Quando fatores de confusão, como o tabagismo e a perda não intencional de peso, são ajustados na análise de taxas de mortalidade, demonstra-se uma relação quase linear entre o Índice de Massa Coporal (IMC) e morte. Quanto maior o período da obesidade, maior é o risco. A obesidade grave está associada com aumento de 12 vezes no risco de mortalidade 
na faixa etária entre 25 e 35 anos, se comparada com pessoas magras ${ }^{3}$. Segundo Karp ${ }^{10}, 25 \%$ das crianças obesas tornar-se-ão adultos obesos, sendo que esta percentagem aumenta para $80 \%$ se a obesidade for na adolescência.

Os estudos relatados pela $\mathrm{OMS}^{3}$ demonstraram que as condições de saúde são afetadas pela obesidade na infância e na adolescência, sendo os distúrbios psicossociais os mais prevalentes, além da persistência da obesidade na vida adulta.

Embora os indicadores epidemiológicos de cárie dentária venham mostrando importantes declínios em sua prevalência, esta morbidade ainda representa o maior problema bucal de saúde pública no Brasil ${ }^{11}$. A mudança de hábitos alimentares, em que os brasileiros vêm substituindo cada vez mais o consumo de alimentos ricos em fibras e nutrientes por alimentos industrializados ricos em gorduras e carboidratos, facilita a instalação da doença ${ }^{12}$, mesmo que os indivíduos estejam expostos a fatores de proteção amplamente difundidos, como os fluoretos.

A relação entre a condição dentária, a função mastigatória e a ingestão de alimentos está bem demonstrada na literatura científica, devido ao reconhecido papel dos açúcares, notadamente a sacarose, na etiologia da doença cárie ${ }^{13}$.

Assim, este artigo tem como objetivo revisar os aspectos gerais da obesidade e da cárie dentária, enfocando a transição alimentar como problema comum ao desenvolvimento de ambas.

\section{Transição alimentar e sua relação com obesidade e cárie dentária}

A transição na alimentação ocorrida neste século, direcionada a uma alimentação mais rica em gorduras, principalmente de origem animal, açúcares e alimentos refinados, com redução do consumo de carboidratos complexos e fibras, tem sido apontada como um fator fundamental para o aumento do número de casos de obesidade. Aliado a estes fatores, a redução da prática de exercícios físicos regulares leva à alteração na composição corporal, aumentando os níveis de gordura ${ }^{14}$. Ainda, a relação dos fatores mencionados com as alterações demográficas, socioeconômicas e epidemiológicas mostra uma redução progressiva da desnutrição e aumento da obesidade ${ }^{15}$. Também, a introdução precoce de alimentos sólidos na alimentação infantil tem sido apontada como fator contribuinte para a obesidade infantil, pois o desmame pode acontecer tão precocemente quanto antes dos quatro meses de idade, mesmo nos casos em que as mães não trabalham fora do domicílio ${ }^{16}$. Este fato foi também observado em um estudo realizado na cidade de Ouro Preto, MG, com 229 crianças de zero a 24 meses de idade. Observou-se que o desmame precoce ocorria aos três meses de idade em $32,2 \%$ dos $\operatorname{casos}^{17}$.

O aleitamento materno tem características protetoras comprovadas em relação ao crescimento sadio nos primeiros anos de vida. Entre suas propriedades importantes relacionadas à imunidade e maior biodisponibilidade de nutrientes, tal como o ferro, pode-se relacionar o seu equilíbrio, qualitativo e quantitativo, na oferta de praticamente todos os nutrientes indispensáveis à alimentação do jovem lactente ${ }^{18}$.

A introdução de líquidos e de outros alimentos, além do leite materno, na faixa etária de três a quatro meses de idade não só é desnecessária, como também pode aumentar o risco da diminuição da produção de leite e de infecções. Além disso, a alimentação precoce utilizando certos tipos de alimentos, como cereais ou vegetais, pode interferir na absorção de ferro, causando deficiências e aumentando o risco, a longo prazo, de anemia, obesidade, hipertensão, arteriosclerose e alergia alimentar ${ }^{19}$.

Pesquisa realizada na cidade de Piracicaba, SP, com 76 crianças entre três e 24 meses de idade, revelou que a prática de desmame precoce estava presente entre o terceiro e quarto mês de vida e que bebidas açucaradas, como chás, foram introduzidas na dieta de grande parte dos lactentes, antes de dois meses de idade, além do 
consumo significativo de refrigerantes durante os 24 meses de idade ${ }^{20}$.

Os dados de desmame precoce confirmaram-se em 337 crianças estudadas no município de São Paulo, SP, o que representava $57,8 \%$ da população estudada. Uma prática alimentar observada em 37,5\% das crianças entre seis e nove meses de idade era o número de mamadeiras recebidas, de quatro a seis por dia. Entre as crianças com idade de nove a 12 meses, $30,0 \%$ recebiam a mesma quantidade de mamadeiras. Esse número de mamadeiras parece excessivo para aquelas crianças que já recebiam duas refeições de sal por dia. Essa situação permite supor que o leite estava sendo consumido, em parte, como complemento das refeições. Destes dados, pode-se observar ainda que a freqüência alimentar observada era de seis a oito vezes por dia e que este fato poderia estar contribuindo, talvez, para um consumo excessivo de alimentos associado a um estímulo à freqüência alimentar excessiva ${ }^{21}$.

Dentre os fatores alimentares, o excesso de energia, principalmente a alta ingestão de lipídeos e carboidratos, favorece o aumento da adiposidade. A freqüência alimentar também é outro aspecto importante, pois indivíduos que consomem maior número de pequenas refeições ao longo do dia apresentam peso relativamente maior do que aqueles que consomem número menor de grandes refeições ${ }^{14}$. A composição alimentar tem importante papel na determinação da obesidade. Embora seja sabido que altos níveis de ingestão energética levam à maior possibilidade de obesidade, estudos seccionais em adultos não demonstraram uma relação direta entre consumo de energia e obesidade ${ }^{22}$. Os dados sugerem, entretanto, que a ingestão de gordura pode estar associada à obesidade, mesmo quando o total de energia ingerida não esteja associado. Demonstrou-se, também, uma associação estatisticamente significativa entre ingestão diária de gorduras saturadas, não-saturadas e carboidratos, dentre eles a sacarose, e a obesidade em crianças.
Estes hábitos alimentares relacionados à obesidade podem também determinar uma maior prevalência de cárie dentária, já que tanto a quantidade de sacarose ingerida quanto a freqüência de ingestão ${ }^{23}$ são importantes fatores envolvidos na etiologia da cárie. A American Dietetic Association ${ }^{24}$ orienta que a nutrição é componente fundamental para a saúde bucal e que tanto a alimentação como a nutrição têm influência direta na progressão da doença cárie.

Karp ${ }^{10}$ relata a existência de uma relação entre cárie dentária e fatores de risco de doenças cardiovasculares em crianças obesas. Obesidade e cárie dentária tendem a aumentar conjuntamente, principalmente devido ao aumento do consumo de açúcar estar relacionado a ambas as situações. Considerando-se que a partir dos seis meses de idade geralmente o leite materno deixa de ser a única fonte de alimentação do bebê, é provável que a introdução precoce de alimentos açucarados, tais como chá, refrigerantes e mel esteja relacionada com a prevalência de cárie dentária nesta faixa etária ${ }^{23}$, estabelecendo um hábito alimentar favorável à obesidade e à cárie dentária.

Diversos estudos têm mostrado o alto consumo de doces, açúcar e chocolates, podendo resultar no aumento da obesidade e da cárie dentária. Rossow et al. ${ }^{25}$ estudaram o padrão de consumo de açúcar na primeira infância em crianças norueguesas. Concluíram que o consumo de açúcar constitui uma dimensão separada dos hábitos alimentares das crianças e que o hábito de consumo é estabelecido já na infância, com padrões de aumento de consumo desde o nascimento até os dois anos de idade. Tal padrão mantém-se durante os cinco primeiros anos de vida. Um estudo sobre associação entre hábitos alimentares e prevalência de cárie, em crianças entre um e dois anos e meio de idade de São Paulo, SP, mostrou uma prevalência significativamente maior entre aquelas que consumiam mamadeiras com leite açucarado do que entre aquelas que consumiam sem a adição de sacarose ${ }^{26}$. Saito et al. ${ }^{27}$ mostraram, em um estudo 
em Piracicaba, SP, que $100 \%$ das crianças de 18 a 48 meses de idade que apresentavam cárie precoce utilizavam mamadeiras com líquidos açucarados.

Game et al. ${ }^{28}$ demonstraram que escolares chilenos consomem doces em uma média de 4,7 vezes por semana, açúcar 6,1 vezes por semana e chocolates 3,9 vezes por semana. Moreira et al. ${ }^{16}$ concluíram, em um estudo sobre hábitos alimentares em Florianópolis, SC, que a maioria das crianças consome salgadinhos, doces, bolachas e leite com achocolatados açucarados, nos lanches entre as refeições. Val et al. ${ }^{29}$ concluíram que escolares de Madrid, Espanha, consomem cerca de 94 gramas de doces e guloseimas por dia e que este consumo excessivo pode levar à diminuição do consumo de outros nutrientes básicos, aumentando a prevalência de enfermidades relacionadas, especialmente cárie dentária e obesidade.

Freeman \& Sheiham ${ }^{30}$ estudaram os fatores que influenciam a decisão dos adolescentes de consumir açúcar. Concluíram que o prazer imediato do paladar açucarado leva ao não reconhecimento dos malefícios associados ao seu consumo. Também a experiência passada de condição de saúde bucal, o nível de educação, juntamente com o comportamento dos pais relacionado ao assunto influenciam na decisão de consumir açúcar.

Peres et al. ${ }^{31}$, ao estudarem aspectos comportamentais e severidade da doença cárie em crianças de Florianópolis, SC, mostraram que aqueles que consumiam produtos açucarados duas a três vezes por dia, todos os dias, apresentavam 4,41 vezes a chance de ter alta severidade de cárie, quando comparadas com as crianças que consumiam estes produtos no máximo uma vez ao dia.

No Brasil, estudo recente mostrou que o consumo de açúcar e refrigerantes participa com $13,7 \%$ da energia consumida pelos indivíduos, caracterizando o aumento no consumo já excessivo de açúcar refinado e refrigerantes como traço marcante e negativo da evolução do padrão alimentar entre 1988 e $1996^{22}$. Salienta-se que este valor está acima do que a $\mathrm{OMS}^{32}$ recomenda como limite populacional máximo para o consumo de açúcar, isto é, 10,0\% do consumo energético total.

\section{CONSIDERAÇÕ ES FINAIS}

Pode-se concluir que a transição alimentar tem importante papel na determinação da obesidade, principalmente se for levado em consideração que a infância é o período em que se estabelece o padrão alimentar. A ingestão de gorduras e carboidratos na alimentação demonstra um estilo de vida determinado pelo comportamento familiar, que está relacionado ao aumento dos níveis de colesterol, obesidade e hipertensão em crianças, além de poder contribuir para o incremento da cárie dentária.

Como a introdução precoce de alimentos sólidos na alimentação infantil pode contribuir para a instalação de um hábito alimentar favorável à obesidade e à cárie dentária, um importante papel dos profissionais de saúde é encorajar a amamentação natural e orientar sobre a não necessidade da introdução precoce de açúcar refinado na mamadeira e em sucos. Os profissionais de saúde devem auxiliar a conscientizar os pais sobre os efeitos imediatos dos hábitos alimentares inapropriados sobre a saúde da criança. Além disso, podem-se utilizar parâmetros bioquímicos anormais como aumento da glicose sangüínea, colesterol, pressão arterial, problemas relacionados ao desenvolvimento ósseo para reforçar as orientações e, desta forma, não centralizar o foco das orientações somente nos riscos relacionados aos problemas de saúde bucal, caracterizando uma estratégia de ação que englobe fatores de risco comuns para a obesidade e cárie dentária.

\section{RE FER Ê N CIAS}

1. World Health Organization. Physical status: the use and interpretation of Anthropometry. Report of a 
WHO Expert Committee. WHO Technical Report Series. Geneva; 1995.

2. Florêncio TMMT, Ferreira HS, França APT, Cavalcante JC, Sawaya AL. Obesity and undernutrition in a very-low-income population in the city of Maceió, Northeastern Brazil. Br J Nutr 2001; 86:277-83.

3. World Health Organization. Obesity: preventing and managing the global epidemic. Report of a WHO consultation on obesity. Geneva; 1998.

4. Eckersley RM. Losing the battle of the bulge: causes and consequences of obesity. Med J Aust 2001; 174:590-2

5. Vischer TL, Seidell JC. The public health impact of obesity. Annu Rev Public Health 2001; 22:355-75.

6. Keller C, Stevens KR. Assessment, etiology, and intervention in obesity in children. Nurse Pract 1996; 21(9):31-41.

7. Monteiro CA, Conde WL. A tendência secular da obesidade segundo estratos sociais: Nordeste e Sudeste do Brasil, 1975-1989-1997. Arq Bras Endocr Metabol 1999; 43(3):186-204.

8. Balaban G, Silva GAP. Prevalência de sobrepeso e obesidade em crianças e adolescentes de uma escola da rede privada de Recife. J Pediatr 2001; 77:96-100.

9. Abrantes MM, Lamounier JA, Colosimo EA. Prevalência de sobrepeso e obesidade em crianças e adolescentes das Regiões Sudeste e Nordeste. J Pediatr 2002; 78(4):335-40.

10. Karp WB. Childhood and adolescent obesity: a national epidemic. Calif Den Assoc J 1998; 26(10):771-3.

11. Traebert J, Peres MA, Galesso E, Zabot NE, Marcenes W. Prevalência e severidade da cárie dentária em escolares de seis e doze anos de idade. Rev Saúde Pública 2001; 35(3):283-8.

12. Mateos A. Brasileiros comem cada vez mais e com pior qualidade. Rev APCD 1999; 53(1):8-20.

13. Krall E, Hayes C, Garcia R. How dentition status and masticatory funcion affect nutrient intake. J Am Dent Assoc 1998; 129(9):1261-9.

14. Francischi RPP, Pereira LO, Freitas CS, Klopfer M, Santos RC, Vieira P, et al. Obesidade: atualização sobre sua etiologia, morbidade e tratamento. Rev Nutr 2000; 13(1):17-28.

15. Monteiro CA, Mondini L, Souza ALM, Popkin BM. Da desnutrição para a obesidade: a transição nutricional no Brasil. In: Monteiro CA. Velhos e novos males da saúde no Brasil: a evolução do país e de suas doenças. São Paulo: Hucitec; 1995.

16. Moreira EAM, Corso ACT, Krüger RM, Rehn A. Desmame precoce e estado nutricional infantil. Rev Ciências Saúde 1996; 15(1-2):126-40.

17. Passos MC, Lamounier JA, Silva CA, Freitas SN, Baudson MF. Práticas de amamentação no município de Ouro Preto, MG, Brasil. Rev Saúde Pública 2000; 4(6):617-22.

18. Yamamoto RM, Sorbata PJP, Neto FJ, Paiva LM, Mizukami MH, Tumas R, Leone C. Tipos de aleitamento e crescimento no primeiro semestre de vida. Pediatria 1995; 17(3):148-54.

19. Horta BL, Olinto MTA, Victora CG, Barros FC, Guimarães PRV. Amamentação e padrões alimentares em crianças de duas coortes de base populacional no Sul do Brasil: tendências e diferenciais. Cad Saúde Pública 1996; 12(Supl 1): 43-8.

20. Tabai KC, Carvalho JF, Salay E. Aleitamento materno e a prática de desmame em duas comunidades rurais de Piracicaba, SP. Rev Nutr 1998; 11(2): 173-83.

21. Souza S, Szarfarc SC, Souza JMP. Prática alimentar no primeiro ano de vida, em crianças atendidas em centros de saúde escola do município de São Paulo. Rev Nutrição 1999; 12(2):167-74.

22. Monteiro CA, Mondini L, Costa RBL. Mudanças na composição e adequação nutricional da dieta familiar em áreas metropolitanas do Brasil (1988-1996). Rev Saúde Pública 2000; 34(3): 251-8

23. Freire MCM. Dieta, saúde bucal e saúde geral. In: Buisch YP. Promoção de saúde bucal na clínica odontológica. São Paulo: Artes Médicas; 2000.

24. American Dietetic Association. Position of the American Dietetic Association: oral health and nutrition. J Am Diet Assoc 1996; 96(2):184-9. 
25. Rossow I, Kjaernes U, Holst D. Patterns of sugar consumption in early childhood. Community Dent Oral Epidemiol 1990; 18:12-6.

26. Mattos-Graner RO, Zelante F, Line RCSR, Mayer MPA. Association between caries prevalence and clinical, microbiological and dietary variables in 1.0 to 2.5-year-old Brazilian children. Caries Res 1998; 32:319-23.

27. Saito SK, Deccico HMU, Santos MN. Efeito da prática de alimentação infantil e de fatores associados sobre a ocorrência da cárie dental em pré-escolares de 18 a 48 meses. Rev Odontol Univ São Paulo 1999; 13(1):5-11.

28. Game JH, Marinchovic RI, Santana MCD, Marinchovic DI. Hábitos alimentares de escolares rurales de la Región Metropolitana de Chile: Un estudio comparativo. Arch Latinoamer Nutr 1996; 46(3):183-9.
29. Val TL, Estivariz CF, Icaya PM, Jaunsolo MA, Olmo D, Martínez CV. Cosumo de alimentos del grupo "dulces y golosinas" en la población infantil escolarizada de la Comunidad Autónoma de Madrid. Med Clin 1997; 109:88-91.

30. Freeman R, Sheiham A. Understanding decisionmaking processes for sugar consumption in adolescence. Community Dent Oral Epidemiol 1997; 25:228-32.

31. Peres KGA, Bastos JRM, Latorre MRDO. Severidade de cárie em crianças e relação com aspectos sociais e comportamentais. Rev Saúde Pública 2000; 34(4):402-8.

32. World Health Organization. Study Group on Diet, nutrition and the prevention of chronic Diseases. Geneva, Report Series. Geneva; 1990.

Recebido para publicação em 4 de novembro de 2002 e aceito em 18 de junho de 2003. 